\title{
Outage Probability of Cooperative Relay Networks in Nakagami- $m$ Fading Channels
}

\author{
Himal A. Suraweera, Student Member, IEEE, Peter J. Smith, Senior Member, IEEE, \\ and Jean Armstrong, Senior Member, IEEE
}

\begin{abstract}
It is well known that the cooperation among nodes can improve the performance of a wireless network. In this letter we analyze the outage probability behaviour of a relay network in Nakagami- $m$ fading channels. A closed-form solution for the outage probability is derived. When $m=1$, the results are applicable for Rayleigh fading. Computer simulations confirm the presented mathematical analysis.
\end{abstract}

Index Terms-Cooperative diversity, multihop transmission, outage probability, Nakagami- $m$ channels.

\section{INTRODUCTION}

$\mathbf{R}$ ECENTLY cooperative and relay protocol strategies have been the subject of great interest among the research community due to their possible use in cellular, ad-hoc/sensor networks and military communications [1]-[3]. These concepts of employing relays to help forward the information to destinations have also been benefited by rapid advancements in the fields of signal processing and low cost microelectronics. As indicated by previous bit error rate or outage probability studies, cooperation techniques among nodes have the high potential to improve the performance of wireless networks [2], [4], [5]. The protocols developed by Laneman and Wornell are capable of exploiting the inherent spatial diversity even if multi-antenna elements are not deployed at the node levels [1]. However concerning relay strategies many communication and information theoretic based problems remain to be answered before encouraging their practical deployment [3], [4].

In [5] Zhao et al. have investigated the outage probability behavior of a decode-and-forward based relay communication system in Rayleigh fading channels. Furthermore, the authors have also provided two tight lower bounds to approximate the outage probability. In this letter, we extend their results and derive the outage probability of the forward-and-decode scheme in independent Nakagami- $m$ fading channels. Recently several authors have investigated the performance of relay networks in Nakagami fading channels [6], [7]. The Nakagami- $m$ model represents a wide variety of realistic lineof-sight/non line-of-site fading channels encountered in practice. Although Nakagami- $m$ and Rician distributions are not

Manuscript received May 31, 2006. The associate editor coordinating the review of this letter and approving it for publication was Prof. Paul Cotae.

H. A. Suraweera and J. Armstrong are with the Department of Electrical and Computer Systems Engineering, PO Box 35, Monash University, Melbourne, Victoria, 3800, Australia (email: \{himal.suraweera, jean.armstrong\}@eng.monash.edu.au).

P. J. Smith is with the Department of Electrical and Computer Engineering, University of Canterbury, Private Bag 4800, Christchurch, New Zealand (email: peter@elec.canterbury.ac.nz).

Digital Object Identifier 10.1109/LCOMM.2006.060834. identical, parameter mappings exist to reduce the difference between their probability density functions (p.d.f). Therefore our solution can be used to investigate the outage probability characteristics under different fading severity conditions. The infinite summation appearing in the outage probability expression can easily be truncated to produce accurate results [8]. By setting $m=1$, our solution can be directly applied to obtain the results of [5]. Numerical and computer simulations confirm our mathematical analysis.

\section{Outage Probability Analysis}

\section{A. System and Channel Model}

We consider a cooperative wireless system with source-todestination, source-to-node, node-to-destination communication links. The number of relay nodes is $a$ and our basic system assumptions are the same as [1], [5]. Furthermore each link is only capable of performing single antenna transmissions.

The fading channel coefficients between source and cooperative nodes are denoted by $h_{s, c}$. Let $h_{s, c}=\left|h_{s, c}\right| e^{j \theta}$ where $\left|h_{s, c}\right|$ is Nakagami distributed with parameters $\hat{m}_{c}, \hat{\Omega}_{c}$ and $\theta$ is assumed to be uniformly distributed over $(0,2 \pi]$. Hence the p.d.f of $\left|h_{s, c}\right|$ is given by

$$
P_{\left|h_{s, c}\right|}(x)=\frac{2}{\Gamma\left(\hat{m}_{c}\right)}\left(\frac{\hat{m}_{c}}{\hat{\Omega}_{c}}\right)^{\hat{m}_{c}} x^{2 \hat{m}_{c}-1} e^{-\frac{\hat{m}_{c}}{\hat{\Omega}_{c}} x^{2}} U(x)
$$

$U(\cdot)$ signifies the unit step function and $\Gamma(\cdot)$ is the gamma function. In the Nakagami- $m$ distribution the parameter $m$ signifies the fading severity and smaller values of $m$ represent more fading in the channel. The amplitudes of the fading channel links between source to destination $\left|h_{s, d}\right|$ and nodes to destination $\left|h_{c, d}\right|$ are also modeled as Nakagami- $m$ variables with parameters $m_{0}, \Omega_{0}$ and $m_{c}, \Omega_{c}$ respectively.

\section{B. Outage Probability}

The mutual information between the source and cooperative nodes $c=1, \ldots, a$ is given by [1]

$$
I_{c}=\frac{1}{a+1} \log _{2}\left(1+\gamma_{t} \hat{x}_{c}\right)
$$

where $\hat{x}_{c}=\left|h_{s, c}\right|^{2}$ follows a Gamma distribution with parameters $\hat{\alpha}_{c}>0$ and $\hat{\beta}_{c}>0$ and $\gamma_{t}$ is the transmitted SNR. The p.d.f of $\hat{x}_{c}$ is given by

$$
p_{\left|h_{s, c}\right|^{2}}(y)=\frac{1}{\Gamma\left(\hat{\alpha}_{c}\right)} \hat{\beta}_{c}^{-\hat{\alpha}_{c}} y^{\hat{\alpha}_{c}-1} e^{-y / \hat{\beta}_{c}} U(y)
$$


where parameters $\hat{\alpha}_{c}$ and $\hat{\beta}_{c}$ are the shape and scale parameters respectively. They are related to the Nakagami- $m$ distribution parameters as $\hat{\alpha}_{c}=\hat{m}_{c}$ and $\hat{\beta}_{c}=\hat{\Omega}_{c} / \hat{m}_{c}$.

The availability of a relay node to assist the source to destination communications, depends on the reliability of that source to relay transmission link. That is when the mutual information of a source-node link becomes greater than the target rate $R$ the node joins the cooperative set $c \in C$ [5]. Hence the mutual information of the decode-and-forward scheme is [1]

$$
I=\frac{1}{a+1} \log _{2}\left(1+\gamma_{t}\left(x_{0}+\sum_{c \in C} x_{c}\right)\right)
$$

where $x_{0}=\left|h_{s, d}\right|^{2}$ and $x_{c}=\left|h_{c, d}\right|^{2}$ are gamma variates with parameters $\left(\alpha_{0}, \beta_{0}\right)$ and $\left(\alpha_{c}, \beta_{c}\right)$ respectively.

The outage probability is one of the most commonly used performance measures in wireless systems [5]. The outage probability mathematically defined as $\mathcal{P}_{o}=P(I<R)$ where $R$ is the predetermined transmission rate of the source. Therefore using the law of total probability $\mathcal{P}_{o}$ for the decodeand forward scheme can be expressed as [5]

$$
\mathcal{P}_{o}=\sum_{C} \mathrm{P}[I<R \mid C] \mathrm{P}[C]
$$

Note that $\mathrm{P}[I<R \mid C=\{1, \ldots, k\}]$ is given by

$$
\mathrm{P}[I<R \mid C=\{1, \ldots, k\}]=\mathrm{P}\left(\sum_{c=0}^{k} x_{c}<\frac{2^{(a+1) R}-1}{\gamma_{t}}\right)
$$

We define $\gamma=\left(2^{(a+1) R}-1\right) / \gamma_{t}$. Before obtaining the cumulative distribution function (CDF) of the random variable $x_{\text {sum }}=\sum_{c=0}^{k} x_{c}$, i.e., sum of several independent Gamma variates, we first state the following result due to Moschopoulos [8], [9].

Result 1: Let $\left\{X_{n}\right\}_{n=1}^{N}$ be independent Gamma variates with parameters $\alpha_{n}$ and $\beta_{n}$ respectively. Then the p.d.f of $Z=X_{1}+X_{2}+\cdots+X_{N}$ can be expressed as

$$
p_{Z}(z)=A \sum_{k=0}^{\infty} \frac{\delta_{k} z^{\sum_{i=1}^{N} \alpha_{i}+k-1} e^{-z / \beta_{1}}}{\Gamma\left(\sum_{i=1}^{N} \alpha_{i}+k\right) \beta_{1}^{\sum_{i=1}^{N} \alpha_{i}+k}} U(z)
$$

where $\beta_{1}=\min _{n}\left\{\beta_{n}\right\}, A=\prod_{i=1}^{N}\left(\beta_{1} / \beta_{i}\right)^{\alpha_{i}}$ and the coefficients $\delta_{k}$ can be obtained from the following recursive relations as

$$
\left\{\begin{array}{l}
\delta_{0}=1 \\
\delta_{k+1}=\frac{1}{k+1} \sum_{i=1}^{k+1}\left(\sum_{j=1}^{N} \alpha_{j}\left(1-\frac{\beta_{1}}{\beta_{j}}\right)^{i}\right) \delta_{k+1-i}
\end{array}\right.
$$

for $k=0,1,2, \ldots$

The Moschopoulos p.d.f representation of the sum of Gamma variables has the advantage of been applicable to any $\left\{\alpha_{n}\right\}_{n=1}^{N}$. That is some $\left\{\alpha_{n}\right\}_{n=1}^{N}$ can be equal while others can be distinct. In the statistical literature, other expressions for $p_{Z}(z)$ in terms of Humbert or Whittaker functions and zonal polynomials exist, see for example [10] and references therein. However the Moschopoulos p.d.f allows us to obtain a mathematically tractable outage probability solution. If all
$N$ scale parameters are equal to $\beta$, the p.d.f simplifies to $Z \sim \operatorname{Gamma}\left(\sum_{i=1}^{N} \alpha_{i}, \beta\right)$ [10]. Since we neglect the path loss effects in this paper, this condition means the shape parameters must also be the same. Note that in the general case, p.d.f of $x_{s}$ can be obtained using (7). The CDF of $x_{s}$ can be calculate from the p.d.f as $F_{\text {sum }}(x)=\int_{-\infty}^{x} p_{\text {sum }}(x) d x$. Hence $F_{\text {sum }}(x)$ can be written as

$$
F_{\text {sum }}(x)=A \sum_{n=0}^{\infty} \delta_{n} \int_{0}^{x} \frac{x^{\sum_{i=1}^{N} \alpha_{i}+n-1} e^{-y / \beta_{1}}}{\Gamma\left(\sum_{i=1}^{N} \alpha_{i}+n\right) \beta_{1}^{\sum_{i=1}^{N} \alpha_{i}+n}} d x
$$

The interchange of the integration and summation above for uniform convergence have been justified in [8] using a rigorous mathematical procedure. Hence for all practical purposes, one can use the first $l$ terms of the series, where $l$ is such that the desired accuracy is obtained. Eq. (9) can be simplified using the results of [11, Sec. 3.381, Eq. 1]. That is $\int_{0}^{u} x^{\nu-1} e^{-\mu x} d x=\mu^{-\nu} \Psi(\nu, \mu u)$ for $\Re\{\nu>0\}$. $\Psi(\cdot, \cdot)$ is the lower incomplete gamma function defined as $\Psi(\kappa, x)=\int_{0}^{x} e^{-t} t^{\kappa-1} d t$. Therefore,

$$
\begin{aligned}
F_{\text {sum }}(x) & =A \sum_{n=0}^{\infty} \delta_{n} \frac{\beta_{1}^{\left(\sum_{i=1}^{N} \alpha_{i}+n\right)}}{\Gamma\left(\sum_{i=1}^{N} \alpha_{i}+n\right) \beta_{1}^{\sum_{i=1}^{N} \alpha_{i}+n}} \\
& \times \Psi\left(\sum_{i=1}^{N} \alpha_{i}+n, \frac{x}{\beta_{1}}\right)
\end{aligned}
$$

Hence using the result of (10) we can now express (6) as

$$
\begin{aligned}
F_{\text {sum }}(\gamma) & =A \sum_{n=0}^{\infty} \delta_{n} \frac{\left(\sum_{i=0}^{k} \alpha_{i}+n\right)^{-1} \gamma\left(\sum_{i=0}^{k} \alpha_{i}+n\right)}{\Gamma\left(\sum_{i=0}^{k} \alpha_{i}+n\right) \beta_{1}^{\sum_{i=0}^{k} \alpha_{i}+n}} \\
& \times{ }_{1} F_{1}\left(\sum_{i=0}^{k} \alpha_{i}+n ; 1+\sum_{i=0}^{k} \alpha_{i}+n ;-\frac{\gamma}{\beta_{1}}\right)
\end{aligned}
$$

where ${ }_{1} F_{1}(a ; b ; z)$ is the confluent hypergeometric function [11]. Note that we have used the identity $\Psi(\alpha, x)=$ $\alpha^{-1} x^{\alpha}{ }_{1} F_{1}(\alpha ; 1+\alpha ;-x)$ to derive $(11) .{ }_{1} F_{1}(\cdot ; \cdot ; \cdot)$ and/or $\Psi(\cdot, \cdot)$ are available in popular symbolic software such as MATHEMATICA and MATLAB.

From the mutual information formula in (2), we get

$$
\mathrm{P}\left[\hat{x}_{c}>\gamma\right]=1-\frac{\hat{\beta}_{c}^{-\hat{\alpha}_{c}} \hat{\alpha}_{c}^{-1} \gamma^{\hat{\alpha}_{c}}}{\Gamma\left(\hat{\alpha}_{c}\right)}{ }_{1} F_{1}\left(\hat{\alpha}_{c} ; 1+\hat{\alpha}_{c} ;-\frac{\gamma}{\hat{\beta}_{c}}\right)
$$

Due the independent channel assumption, the probability that $k$ nodes cooperate in relaying information to the destination can be easily calculated. $\mathrm{P}[|C|=k]$ can be obtained by summing probability terms $\mathrm{P}_{\mathcal{K}}$ over all node permutations. As an example, for a given node permutation $\mathcal{K}$ we can write

$$
\mathrm{P}_{\mathcal{K}}=\prod_{l=1}^{k} \mathrm{P}_{l}\left(\hat{x}_{l}>\gamma\right) \prod_{r=1, r \neq l}^{a-l}\left(1-\mathrm{P}_{r}\left(\hat{x}_{r}>\gamma\right)\right.
$$

and $\mathrm{P}[|C|=k]=\sum_{\mathcal{K}} \mathrm{P}_{\mathcal{K}}$. Note that in (13) if we assume identical Nakagami fading conditions for all source-node links, then (13) simplifies as [5]

$$
\mathrm{P}[|C|=k]=\left(\begin{array}{l}
a \\
k
\end{array}\right)\left(\mathrm{P}\left[\hat{x}_{c}>\gamma\right]\right)^{k}\left(1-\mathrm{P}\left[\hat{x}_{c}>\gamma\right]\right)^{a-k}
$$




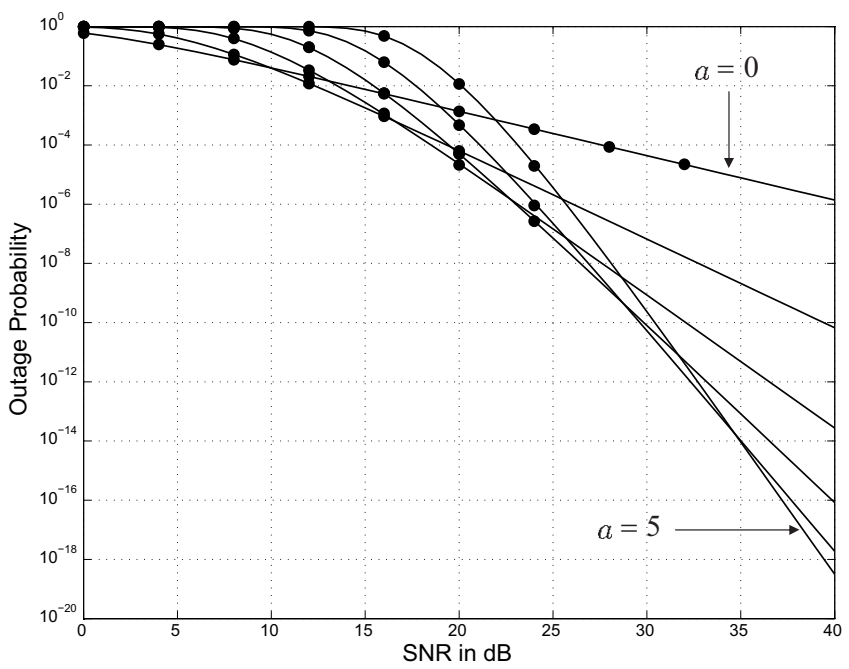

Fig. 1. Outage probability against the transmitted SNR for different number of cooperative nodes. The analytical results are due to (15). Simulations are shown with dots.

Finally the outage probability of the system is given by

$$
\begin{aligned}
\mathrm{P}[I<R] & =\sum_{k=0}^{a} \mathrm{P}[I<R|| C \mid=k] \mathrm{P}[|C|=k] \\
& =\sum_{k=0}^{a} F_{\text {sum }}(\gamma) \mathrm{P}[|C|=k]
\end{aligned}
$$

In the next Section we have presented some numerical and simulation results to confirm the theoretical analysis.

\section{Simulations AND Numerical Results}

MATLAB was used to plot all simulation and theoretical results. In the simulations we have assumed equal average fading power for all source-cooperative, source-destination and cooperative-destination links. That is $\hat{\Omega}_{c}=\Omega_{c}=\Omega_{0}=1$. We set $R=1 \mathrm{bit} / \mathrm{s} / \mathrm{Hz}$. Fig. 1 shows the outage probability of a decode-and-forward system with the number of relay nodes ranging from 0 to 5 . For all links, $m=1.5$. As Fig. 1 shows depending on the SNR, the optimum number of relay nodes vary. Only in the very high SNR regime, $a=5$ outperforms. This is due to the time sharing nature of the protocol reflected in mutual information expression by the factor $1 /(a+1)$. In SNR regions, although large number of relays indicates a higher diversity order, values for mutual information seem to be governed by the SNR. Therefore dividing by the term $1 /(a+1)$ impacts the outage performance.

Fig. 2 shows outage probability plots of the network for different $m$. To compare, we have also included the plot with $m=1$. With $m=1$, the Nakagami- $m$ model represents Rayleigh fading. Simulation results are also provided to confirm the theoretical analysis. Although for SNR $<10$ $\mathrm{dB}$ the outage performance appears same, when $m>1$ as Fig. 2 illustrates compared to Rayleigh fading Nakagami- $m$ channels can deliver higher order diversity gains. Our theoretical analysis applies to non identically distributed channels, therefore impact of path loss effects and different fading severity scenarios on outage probability can also be studied.

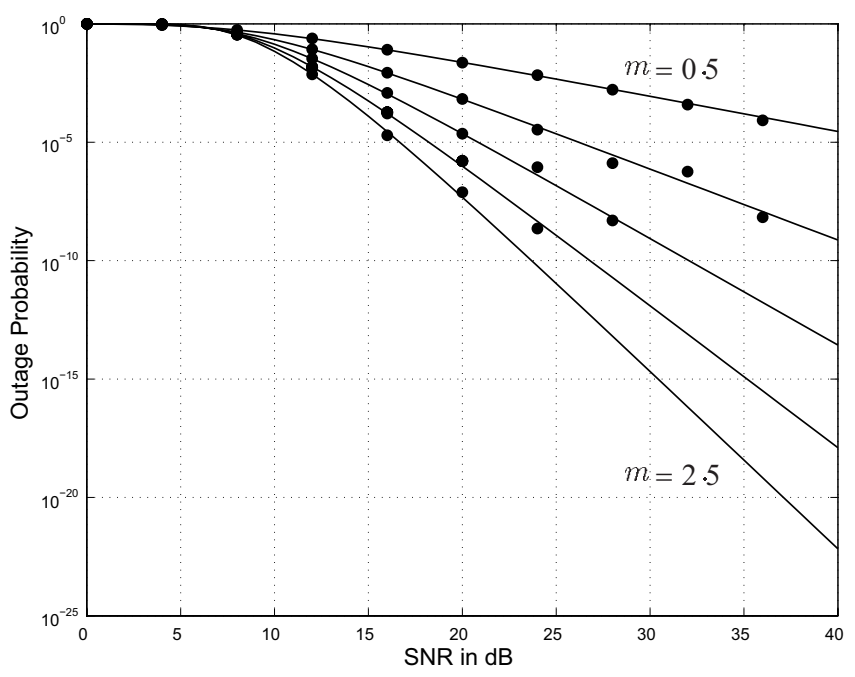

Fig. 2. Outage probability against the transmitted SNR for $m=0.5,1,1.5$, 2 , and 2.5. Simulations are shown with dots. In all cases $a=2$.

\section{CONCLUding Remarks}

We have analyzed the outage probability of a wireless relay network over Nakagami- $m$ fading channels. The theoretical outage probability have been derived by using results appearing in statistical literature. The solution is helpful to investigate the outage probability of cooperative networks under different fading severity conditions.

\section{REFERENCES}

[1] J. N. Laneman and G. W. Wornell, "Distributed space-time coded protocols for exploiting cooperative diversity in wireless networks," IEEE Trans. Inf. Theory, vol. 49, pp. 2415-2425, Nov. 2003.

[2] J. Boyer, D. D. Falconer, and H. Yanikomeroglu, "Multihop diversity in wireless relaying channels," IEEE Trans. Commun., vol. 52, pp. 18201830, Oct. 2004.

[3] A. Nosratinia, T. E. Hunter, and A. Hedayat, "Cooperative communication in wireless networks," IEEE Commun. Mag., vol. 42, pp. 74-80, Oct. 2004.

[4] Y. Fan and J. S. Thompson, "On the outage capacity of MIMO multihop networks," in Proc. IEEE GLOBECOM '05, pp. 2204-2208.

[5] Y. Zhao, R. Adve, and T. J. Lim, "Outage probability at arbitrary SNR with cooperative diversity," IEEE Commun. Lett., vol. 9, pp. 700-702, Aug. 2005.

[6] M. O. Hasna and M.-S. Alouini, "Outage probability of multihop transmission over Nakagami fading channels," IEEE Commun. Lett., vol. 7, pp. 216-218, May 2003.

[7] G. K. Karagiannidis, T. A. Tsiftsis, and R. K. Mallik, "Bounds for multihop relayed communications in Nakagami- $m$ fading," IEEE Trans. Commun., vol. 54, pp. 18-22, Jan. 2006

[8] P. G. Moschopoulos, "The distribution of the sum of independent Gamma random variables," Ann. Inst. Statist. Math. (Part A), vol. 37, pp. 541-543, 1985.

[9] M.-S. Alouini, A. Abdi, and M. Kaveh, "Sum of Gamma variates and performance of wireless communication systems over Nakagami-fading channels," IEEE Trans. Veh. Technol., vol. 50, pp. 1471-1479, Nov. 2001.

[10] T. Pham-Gia and N. Turkkan, "System availability in a gamma alternating renewal process," Naval Research Logistics, vol. 46, pp. 832-844, 1999.

[11] I. S. Gradshteyn and I. M. Ryzhik, Table of Integrals, Series and Products. San Diego: CA, Academic Press, 1980. 